\title{
Relativistic beaming and gamma-ray brightness of blazars
}

\author{
T. Savolainen ${ }^{1}$, D. C. Homan ${ }^{2}$, T. Hovatta ${ }^{3,4}$, M. Kadler ${ }^{5,6,7}$, Y. Y. Kovalev ${ }^{8,1}$, M. L. Lister ${ }^{3}$, E. Ros ${ }^{1,9}$, and J. A. Zensus ${ }^{1}$
}

1 Max-Planck-Institut für Radioastronomie, Auf dem Hügel 69, 53121 Bonn, Germany

e-mail: tsavolainen@mpifr-bonn.mpg.de

2 Department of Physics and Astronomy, Denison University, Granville, OH 43023, USA

3 Department of Physics, Purdue University, 525 Northwestern Avenue, West Lafayette, IN 47907, USA

4 Metsähovi Radio Observatory, Helsinki University of Technology TKK, Metsähovintie 114, 02540 Kylmälä, Finland

5 Dr. Karl Remeis-Observatory \& ECAP, Friedrich-Alexander University Erlangen-Nuremberg, Sternwartstr. 7, 96049 Bamberg, Germany

${ }^{6}$ CRESST/NASA Goddard Space Flight Center, Greenbelt, MD 20771, USA

7 Universities Space Research Association, 10211 Wincopin Circle, Suite 500 Columbia, MD 21044, USA

8 Astro Space Center of Lebedev Physical Institute, Profsoyuznaya 84/32, 117997 Moscow, Russia

9 Departament d'Astronomia i Astrofísica, Universitat de València, 46100 Burjassot, Valencia, Spain

Received 25 November 2009 / Accepted 12 January 2010

\section{ABSTRACT}

\begin{abstract}
Aims. We investigate the dependence of $\gamma$-ray brightness of blazars on intrinsic properties of their parsec-scale radio jets and the implication for relativistic beaming.

Methods. By combining apparent jet speeds derived from high-resolution VLBA images from the MOJAVE program with millimetrewavelength flux density monitoring data from Metsähovi Radio Observatory, we estimate the jet Doppler factors, Lorentz factors, and viewing angles for a sample of 62 blazars. We study the trends in these quantities between the sources which were detected in $\gamma$-rays by the Fermi Large Area Telescope (LAT) during its first three months of science operations and those which were not detected. Results. The LAT-detected blazars have on average higher Doppler factors than non-LAT-detected blazars, as has been implied indirectly in several earlier studies. We find statistically significant differences in the viewing angle distributions between $\gamma$-ray bright and weak sources. Most interestingly, $\gamma$-ray bright blazars have a distribution of comoving frame viewing angles that is significantly narrower than that of $\gamma$-ray weak blazars and centred roughly perpendicular to the jet axis. The lack of $\gamma$-ray bright blazars at large comoving frame viewing angles can be explained by relativistic beaming of $\gamma$-rays, while the apparent lack of $\gamma$-ray bright blazars at small comoving frame viewing angles, if confirmed with larger samples, may suggest an intrinsic anisotropy or Lorentz factor dependence of the $\gamma$-ray emission.
\end{abstract}

Key words. galaxies: active - galaxies: jets - quasars: general - BL Lacertae objects: general

\section{Introduction}

One of the most important discoveries of the Energetic GammaRay Experiment Telescope (EGRET) on-board the Compton Gamma-Ray Observatory in the 1990s was the detection of over 65 active galactic nuclei (AGN) at photon energies above $100 \mathrm{MeV}$ (Hartman et al. 1999; Mattox et al. 2001). The detected sources were almost exclusively blazars, a class of highly variable AGN comprised of flat spectrum radio quasars and BL Lac objects. The distinctive characteristic of blazars is a relativistic jet oriented close to our line-of-sight. Synchrotron radiation of energetic electrons in the jet dominates the low energy end of the blazar spectral energy distribution. This emission is strongly beamed due to relativistic effects which increase the observed flux density of a stationary jet by a factor of $\delta^{2-\alpha}$ and that of distinct "blobs" in the jet by a factor of $\delta^{3-\alpha}$. Here $\delta$ is the jet Doppler factor and $\alpha$ is the spectral index defined as $S_{v} \propto v^{+\alpha}$ (Blandford \& Königl 1979). The Doppler factor is defined as $\delta=[\Gamma(1-\beta \cos \theta)]^{-1}$, where $\Gamma=\left(1-\beta^{2}\right)^{-1 / 2}$ is the bulk Lorentz factor, $\beta$ is the jet speed divided by the speed of light, and $\theta$ is the angle between the jet and our line-of-sight. The requirement that the $\gamma$-ray-bright sources are transparent to $\gamma \gamma$ pair production, together with their small sizes deduced from the fast $\gamma$-ray variability, strongly suggest that the $\gamma$-ray emission originates in the jet and is also relativistically beamed, in the same manner as the radio emission (von Montigny et al. 1995; Mattox et al. 1993). The many correlations found between the $\gamma$-ray emission detected by EGRET and the radio/mm-wave properties of blazars further support this scenario (Valtaoja \& Teräsranta 1995; Jorstad et al. 2001a,b; Lähteenmäki \& Valtaoja 2003; Kellermann et al. 2004; Kovalev et al. 2005). Since the $\gamma$-ray spectrum is typically steeper than the radio spectrum (Abdo et al. 2009b), it is possible that the $\gamma$-ray emission is even more enhanced by Doppler boosting than the radio emission.

Although inverse Compton (IC) scattering of soft photons off relativistic electrons in the jet is currently the favoured model for the $\gamma$-ray emission, there is a substantial controversy within this model about the origin of the target photon field and the location of the emission site. The seed photons could be, for example, synchrotron photons emitted by the same electrons which scatter them later, (synchrotron self-Compton model, SSC; Maraschi et al. 1992; Bloom \& Marscher 1996) or synchrotron photons emitted by electrons in a different layer of the jet (Ghisellini et al. 2005). The seed photons can also originate in sources external to the jet like the accretion disk, the broad line region clouds or the dust torus (external Compton model; Dermer et al. 1992; Ghisellini \& Madau 1996; Sikora et al. 1994; Błażejowski et al. 2000). Beside these leptonic models there are also a number of 
models where $\gamma$-rays are produced by hadronic processes initiated by relativistic protons co-accelerated with electrons (e.g., Mannheim 1993).

The Large Area Telescope (LAT) on-board the Fermi Gamma-ray Space Telescope is a successor to EGRET, with much better sensitivity, larger energy range (up to $300 \mathrm{GeV}$ ), better angular resolution, and larger field-of-view (Atwood et al. 2009). In only its first three months of science operations, the LAT detected 205 bright $\gamma$-ray sources at $>10 \sigma$ level (Abdo et al. 2009a), 116 of which are associated with AGN at high galactic latitudes $\left(|b| \geq 10^{\circ}\right.$ ) (Abdo et al. 2009b). Here we refer to these 116 sources as "LAT-detected sources". Based on the longterm monitoring of the radio jet motions with the Very Long Baseline Array (VLBA), it was recently shown that the LATdetected quasars have significantly faster apparent jet speeds and higher core brightness temperatures than non-LAT-detected quasars (Lister et al. 2009c; Kovalev et al. 2009). The LAT $\gamma$-ray photon flux also correlates with the compact radio flux density and the flares in $\gamma$-rays and radio seem to happen in the VLBI cores within a typical apparent time separation of up to several months (Kovalev et al. 2009). The $\gamma$-ray bright blazars also have larger-than-average apparent jet opening angles (Pushkarev et al. 2009). These findings indicate that $\gamma$-ray bright blazars are likely more Doppler boosted than $\gamma$-ray faint ones, but what remains unknown is a possible dependence of intrinsic $\gamma$-ray luminosity on parsec-scale jet properties such as the bulk Lorentz factor or the viewing angle in the comoving frame of the jet (Lister et al. 2009c). In this paper we confirm the connection between the $\gamma$-ray brightness and the Doppler factor of the parsec-scale jet for a sample of 62 blazars. We have also combined measurements of the apparent jet speeds and temporal variability at mmwavelengths to derive jet Lorentz factor and the viewing angle in the observer's frame and in the frame comoving with the jet for 57 blazars in order to study how the $\gamma$-ray detection probability depends on these intrinsic jet parameters.

\section{Sample definition and data}

The MOJAVE program, together with its predecessor, the VLBA $2 \mathrm{~cm}$ Survey, has continuously monitored the structural changes in the parsec-scale jets of the compact extragalactic radio sources with the VLBA since 1994 (Kellermann et al. 2004; Lister et al. 2009a). The program provides accurate measurements of the apparent jet speeds, which are typically superluminal (Lister et al. 2009b). The current monitoring list includes a statistically complete, flux-density-limited sample of all 135 sources that lie above $\mathrm{J} 2000$ declination $-20^{\circ}$ and had a $15 \mathrm{GHz}$ compact flux density larger than $1.5 \mathrm{Jy}$ ( $2 \mathrm{Jy}$ for sources below declination of $0^{\circ}$ ) at any time between 1994.0 and 2004.0. Since the sample is selected based on the relativistically beamed, compact jet emission, it mostly comprises blazars and thus can be compared with the sample of $\gamma$-ray-loud AGN.

The Metsähovi Radio Observatory in Finland has regularly monitored the flux density variability of bright northern hemisphere blazars at 22 and $37 \mathrm{GHz}$ since the early 1980s, and decade or longer flux density curves exist for $\sim 100$ sources, which roughly approximate a flux-density-limited sample at $22 \mathrm{GHz}$ (Teräsranta et al. 2004). From these data Hovatta et al. (2009) were able to estimate the Doppler beaming factors of 87 sources by applying a light-travel time argument. If one assumes that the variability timescale of the mm-wave flares seen in these sources corresponds to the light-travel time across the emission region (Jorstad et al. 2005), and that the intrinsic brightness temperature of the source is limited to the equipartition value, $T_{\mathrm{b} \text {, int }} \lesssim 5 \times 10^{10} \mathrm{~K}$ (Readhead 1994), measuring the flare timescale and amplitude provides a way to estimate the variability Doppler factor, $\delta_{\text {var }}$. By combining Eqs. (2) and (3) in Hovatta et al. (2009) one gets

$\delta_{\mathrm{var}}=\left(1.47 \times 10^{13} \frac{\Delta S_{\max } d_{\mathrm{L}}^{2}}{v^{2} \Delta t^{2}(1+z) T_{\mathrm{b}, \text { int }}}\right)^{1 / 3}$,

where $\Delta S_{\max }$ is the flare amplitude in Janskys, $v$ is the observing frequency in $\mathrm{GHz}, \Delta t$ is the timescale of the fastest flare in the source in days, $d_{\mathrm{L}}$ is the luminosity distance in Mpc, and $z$ is the redshift. This method of estimating $\delta_{\text {var }}$ relies on the assumption that every source occasionally reaches $T_{\mathrm{b} \text {,int }}=5 \times 10^{10} \mathrm{~K}$, that the Doppler factor is constant with time, and that the flux density curve at mm-wavelengths is a superposition of flares with mixed duration and amplitude (Hovatta et al. 2009). Thus, identifying the sharpest, fastest flare over a long period of time gives the best estimate of the true Doppler factor. We note that there is an inherent limit to the maximum observable $\delta_{\text {var }}$ of about $40-50$ due to the time sampling of Metsähovi flux curves. We have carried out Monte Carlo simulations, however, which show that this limit does not affect the statistical results reported in this paper.

Of the 87 sources in Hovatta et al. (2009), 60 are blazars that belong to the flux-density-limited MOJAVE sample, have reliable redshifts, and are at galactic latitude $|b| \geq 10^{\circ}$. In this paper, we refer to them as the Metsähovi-MOJAVE (MM) sample. Two additional sources, the BL Lac object B0109+224 and the quasar B1334-127, which were not originally listed in Hovatta et al. (2009), were also included in the sample since new data allowed determination of their $\delta_{\mathrm{var}}$. With these additions, the sample contains 48 quasars and 14 BL Lac objects.

There are $23(37 \%)$ sources in the MM sample that are associated with bright LAT-detected $\gamma$-ray sources (Abdo et al. 2009b): 15 quasars (31\% of the quasars in the MM sample) and 8 BL Lacs (57\% of the BL Lacs). The fraction of LATdetected sources in the MM sample (37\%) is slightly higher than in the full flux-density-limited MOJAVE sample (24\%) (Lister et al. 2009c), but the difference is not statistically significant. An Anderson-Darling (A-D) variant of the Kolmogorov-Smirnov test (Press et al. 1992) does not show a significant difference between the redshift distributions of LAT-detected and non-LATdetected sources in the MM sample. For all the statistical tests reported in this paper, we adopt a significance level of 0.05 for rejecting the null hypothesis. We use the non-parametric A-D test throughout as a test for similarity of two observed distributions, since it is more sensitive to differences in the distribution tails than the original Kolmogorov-Smirnov test.

\section{Results}

\subsection{Doppler factor and Lorentz factor distributions}

The distributions of the variability Doppler factor for the nonLAT-detected and the LAT-detected blazars in the MM sample are shown in the upper left panel of Fig. 1, and the numerical data are listed in Table 1. The LAT-detected sources have on average significantly higher $\delta_{\text {var }}$ values than non-LATdetected sources: the mean $\delta_{\text {var }}$ is $17.1 \pm 1.6$ for the former and $12.2 \pm 1.2$ for the latter. For quasars only, the mean $\delta_{\text {var }}$ are $19.8 \pm 1.7$ for LAT-detected and $13.4 \pm 1.3$ for non-LAT-detected, and there are no LAT-detected quasars with $\delta_{\text {var }}<10$. A onesided Student's t-test confirms that the LAT-detected sources have a higher mean $\delta_{\text {var }}$ than the non-LAT-detected ones with the probability of $99.3 \%$ for blazars, and $99.7 \%$ for quasars only. 
T. Savolainen et al.: Relativistic beaming and gamma-ray brightness of blazars
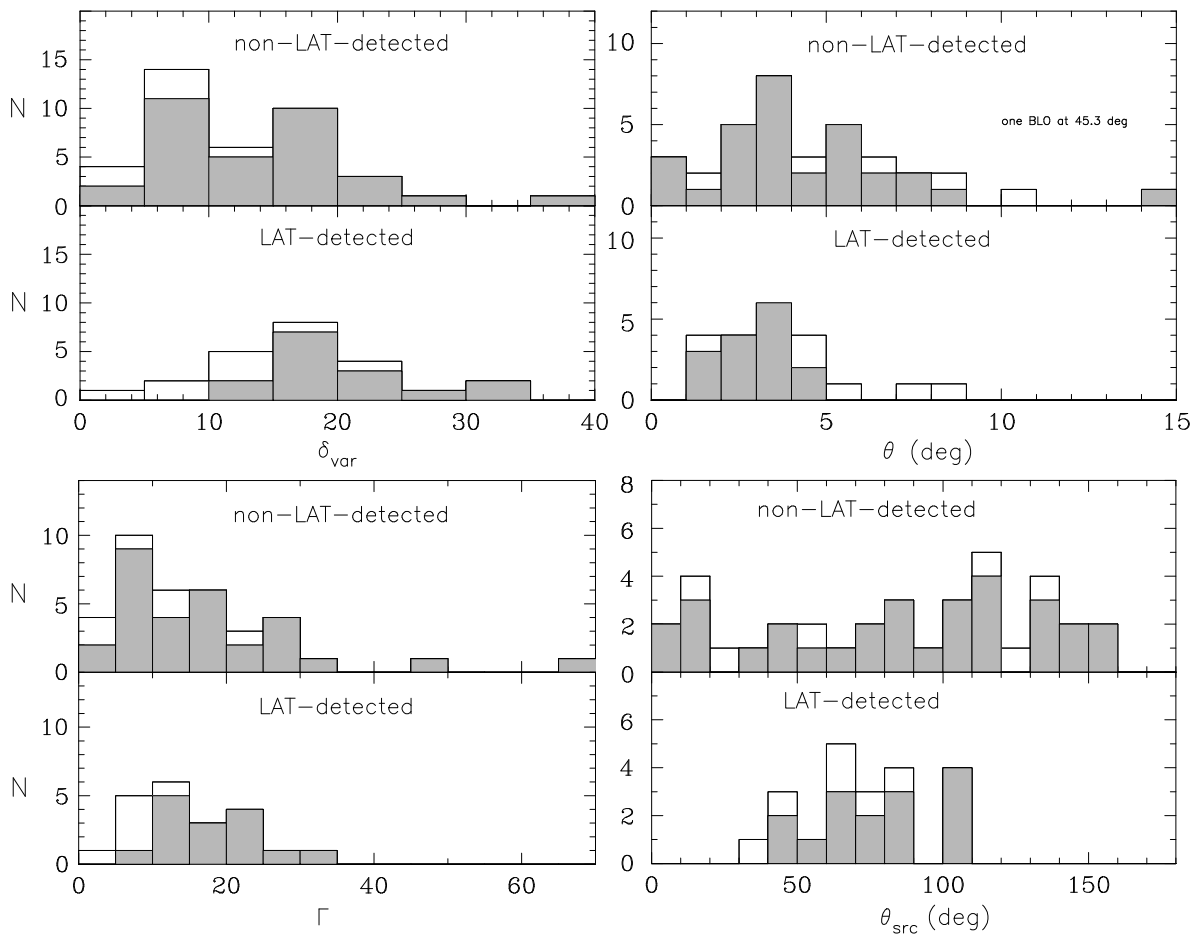

Fig. 1. Upper left: variability Doppler factor $\left(\delta_{\text {var }}\right)$ distributions for non-LAT-detected (upper sub-panel) and LAT-detected (lower subpanel) blazars in the Metsähovi-MOJAVE sample. Quasars are denoted by shaded bins. Lower left: bulk Lorentz factor $(\Gamma)$ distributions. Upper right: distributions of the angles between the jet and our line-of-sight in the observer's frame $(\theta)$. Lower right: distributions of the viewing angles in the comoving frame of the jet $\left(\theta_{\text {src }}\right)$. The values were calculated assuming $\Lambda \mathrm{CDM}$ cosmology with $H_{0}=$ $71 \mathrm{~km} \mathrm{~s}^{-1} \mathrm{Mpc}^{-1}, \Omega_{\mathrm{M}}=0.27$, and $\Omega_{\Lambda}=0.73$.
Since the apparent jet speed $\left(\beta_{\mathrm{app}}\right)$ and the Doppler factor both depend on the bulk Lorentz factor and the viewing angle of the jet, it is possible to calculate the latter quantities if the measured $\delta_{\text {var }}$ and $\beta_{\text {app }}$ both correspond to the same underlying flow speed (Hovatta et al. 2009):

$\Gamma=\frac{\beta_{\mathrm{app}}^{2}+\delta_{\mathrm{var}}^{2}+1}{2 \delta_{\mathrm{var}}}, \quad \theta=\arctan \left(\frac{2 \beta_{\mathrm{app}}}{\beta_{\mathrm{app}}^{2}+\delta_{\mathrm{var}}^{2}-1}\right)$.

Using the data gathered in the MOJAVE program, we have measured $\beta_{\text {app }}$ for 57 out of 62 sources in the MM sample by tracking the motion of the bright features in the jet (Lister et al. 2009b). As shown by Lister et al. (2009b), there is a characteristic flow speed for each jet that can be traced by $\beta_{\text {app }}$. We consider the fastest, non-accelerating, radially moving component to be the most representative of the speed of the underlying flow close to the jet core, where the mm-wavelength outbursts take place (Savolainen et al. 2002). The speeds are listed in Table 1 together with the calculated jet properties. The Lorentz factor distributions for non-LAT-detected and LAT-detected blazars are shown in the lower left panel of Fig. 1. Although there seems to be a lack of LAT-detected quasars with small Lorentz factors (there are no LAT-detected quasars with $\Gamma<8$ ), an A-D test does not detect a statistically significant difference between the distributions - neither for blazars nor for quasars alone.

\subsection{Viewing angle distributions}

The (observer's frame) viewing angle distributions are plotted in the upper right panel of Fig. 1. The $\gamma$-ray bright sources seem to have a narrower $\theta$-distribution than $\gamma$-ray weak sources. The LAT-detected quasars all have $1^{\circ}<\theta<5^{\circ}$, while a significantly wider range of values is covered for the non-LATdetected quasars: the observed $\theta$-distributions of LAT-detected and non-LAT-detected quasars have a probability of $p=0.04$ of being drawn from the same parent distribution, according to an A-D test. For the whole blazar sample, the difference is not statistically significant. The mean value of $\theta$ is slightly smaller for the LAT-detected quasars $\left(2.9 \pm 0.3^{\circ}\right)$ than for the non-LATdetected ones $\left(4.4 \pm 0.6^{\circ}\right)$, but this difference is not statistically significant ( $p=0.06$ according to a Student's t-test).

Because of relativistic aberration, the photons arriving to us at an angle $\theta$ with respect to the jet flow direction were emitted from the jet at an angle $\theta_{\text {src }}$ in the frame comoving with the jet:

$\theta_{\mathrm{src}}=\arccos \left(\frac{\cos \theta-\beta}{1-\beta \cos \theta}\right)$.

The distributions of viewing angles in the comoving frame are shown in the lower right panel of Fig. 1, where we see that the $\theta_{\text {src }}$-distribution is much narrower for the LAT-detected blazars than for the non-LAT-detected blazars. The comoving fluid frame viewing angles of $\gamma$-ray bright blazars are confined to a range between $30^{\circ}$ and $110^{\circ}$ with a median value of $\sim 75^{\circ}$, while the $\theta_{\text {src }}$-distribution of $\gamma$-ray weak blazars ranges from $0^{\circ}$ to $160^{\circ}$. Both small and large values of $\theta_{\text {src }}$ are missing from the LAT-detected sample. An A-D test gives a probability of $p=0.02$ that both samples are derived from the same parent distribution and rejects the null hypothesis. For the smaller sample of quasars only, an A-D test gives $p=0.09$ and does not reject the null hypothesis.

\section{Discussion}

The variability Doppler factors from Hovatta et al. (2009) were determined over a multi-year monitoring program while the $\gamma$-ray detections used in our analysis are based on just three-months of LAT monitoring. The sharp distinction seen in the $\delta_{\text {var }}$ values between the LAT-detected and non-LATdetected sources strongly supports the idea that $\delta_{\text {var }}$ must remain fairly constant with time. Since the LAT-detected and non-LATdetected sources are treated in the exact same way with respect to the derivation of their $\delta_{\mathrm{var}}$, any temporal variation in the Doppler factor (or in $T_{\mathrm{b}, \text { int }}$ ) would thus only serve to destroy the possible correlation and could not create one. Therefore, we consider the 
A\&A 512, A24 (2010)

Table 1. Jet properties of the blazars in the Metsähovi-MOJAVE sample.

\begin{tabular}{|c|c|c|c|c|c|c|c|c|}
\hline $\begin{array}{l}\text { IAU name } \\
\text { (B1950.0) }\end{array}$ & Class & $z$ & $\beta_{\text {app }}^{1}$ & $\delta_{\mathrm{var}}$ & $\Gamma$ & $\begin{array}{c}\theta \\
(\operatorname{deg})\end{array}$ & $\begin{array}{c}\theta_{\mathrm{src}} \\
(\mathrm{deg})\end{array}$ & LAT-det. $^{2}$ \\
\hline $0003-066$ & BL Lac & 0.347 & 0.9 & 5.1 & 2.7 & 4.0 & 20.5 & $\mathrm{~N}$ \\
\hline $0016+731$ & Quasar & 1.781 & 6.7 & 7.8 & 6.9 & 7.3 & 82.0 & $\mathrm{~N}$ \\
\hline $0106+013$ & Quasar & 2.099 & 26.5 & 18.2 & 28.4 & 2.9 & 111.0 & $\mathrm{~N}$ \\
\hline $0109+224$ & BL Lac & 0.265 & $\ldots$ & 9.1 & $\ldots$ & $\ldots$ & $\ldots$ & $\mathrm{Y}$ \\
\hline $0133+476$ & Quasar & 0.859 & 13.0 & 20.5 & 14.4 & 2.5 & 64.8 & $\mathrm{Y}$ \\
\hline $0202+149$ & Quasar & 0.405 & 6.4 & 15.0 & 8.9 & 2.8 & 46.6 & $\mathrm{~N}$ \\
\hline $0212+735$ & Quasar & 2.367 & 7.6 & 8.4 & 7.7 & 6.8 & 84.9 & $\mathrm{~N}$ \\
\hline $0234+285$ & Quasar & 1.207 & 12.3 & 16.0 & 12.7 & 3.5 & 75.4 & $\mathrm{Y}$ \\
\hline $0235+164$ & BL Lac & 0.940 & $\ldots$ & 23.8 & $\ldots$ & $\ldots$ & $\ldots$ & $\mathrm{Y}$ \\
\hline $0333+321$ & Quasar & 1.259 & 12.8 & 22.0 & 14.7 & 2.3 & 60.5 & $\mathrm{~N}$ \\
\hline 0336-019 & Quasar & 0.852 & 22.4 & 17.2 & 23.2 & 3.2 & 104.9 & $\mathrm{~N}$ \\
\hline 0420-014 & Quasar & 0.915 & 7.3 & 19.7 & 11.3 & 1.9 & 41.0 & $\mathrm{Y}$ \\
\hline $0458-020$ & Quasar & 2.286 & 16.5 & 15.7 & 16.6 & 3.7 & 93.1 & $\mathrm{~N}$ \\
\hline $0528+134$ & Quasar & 2.070 & 19.2 & 30.9 & 21.4 & 1.7 & 63.7 & $\mathrm{Y}$ \\
\hline $0605-085$ & Quasar & 0.872 & 16.8 & 7.5 & 22.6 & 5.7 & 131.8 & $\mathrm{~N}$ \\
\hline $0642+449$ & Quasar & 3.396 & 0.8 & 10.6 & 5.4 & 0.8 & 8.3 & $\mathrm{~N}$ \\
\hline $0716+714$ & BL Lac & 0.310 & 10.1 & 10.8 & 10.2 & 5.3 & 86.4 & $\mathrm{Y}$ \\
\hline $0736+017$ & Quasar & 0.191 & 14.4 & 8.5 & 16.5 & 5.9 & 119.0 & $\mathrm{~N}$ \\
\hline $0754+100$ & BL Lac & 0.266 & 14.4 & 5.5 & 21.6 & 6.9 & 138.0 & $\mathrm{~N}$ \\
\hline $0804+499$ & Quasar & 1.436 & 1.8 & 35.2 & 17.6 & 0.2 & 6.0 & $\mathrm{~N}$ \\
\hline $0814+425$ & BL Lac & 0.245 & 1.7 & 4.6 & 2.7 & 8.6 & 42.8 & $\mathrm{Y}$ \\
\hline $0827+243$ & Quasar & 0.941 & 22.0 & 13.0 & 25.2 & 3.9 & 119.0 & $\mathrm{~N}$ \\
\hline $0836+710$ & Quasar & 2.218 & 25.4 & 16.1 & 28.1 & 3.2 & 115.2 & $\mathrm{~N}$ \\
\hline $0851+202$ & BL Lac & 0.306 & 5.2 & 16.8 & 9.3 & 1.9 & 34.5 & $\mathrm{Y}$ \\
\hline $0923+392$ & Quasar & 0.695 & 0.6 & 4.3 & 2.3 & 3.9 & 16.7 & $\mathrm{~N}$ \\
\hline $0945+408$ & Quasar & 1.249 & 18.6 & 6.3 & 30.5 & 5.5 & 142.4 & $\mathrm{~N}$ \\
\hline $1055+018$ & Quasar & 0.888 & 8.1 & 12.1 & 8.8 & 4.4 & 67.6 & $\mathrm{Y}$ \\
\hline $1156+295$ & Quasar & 0.729 & 24.9 & 28.2 & 25.1 & 2.0 & 82.9 & $\mathrm{Y}$ \\
\hline $1222+216$ & Quasar & 0.432 & 21.0 & 5.2 & 45.5 & 5.1 & 152.5 & $\mathrm{~N}$ \\
\hline $1226+023$ & Quasar & 0.158 & 13.4 & 16.8 & 13.8 & 3.3 & 77.1 & $\mathrm{Y}$ \\
\hline $1253-055$ & Quasar & 0.536 & 20.6 & 23.8 & 20.8 & 2.4 & 81.9 & $\mathrm{Y}$ \\
\hline $1308+326$ & Quasar & 0.997 & 20.9 & 15.3 & 22.0 & 3.6 & 107.8 & $\mathrm{Y}$ \\
\hline $1324+224$ & Quasar & 1.400 & & 21.0 & $\ldots$ & $\ldots$ & $\ldots$ & $\mathrm{N}$ \\
\hline $1334-127$ & Quasar & 0.539 & 10.3 & 8.3 & 10.6 & 6.8 & 102.6 & $\mathrm{~N}$ \\
\hline $1413+135$ & BL Lac & 0.247 & 1.8 & 12.1 & 6.2 & 1.4 & 17.1 & $\mathrm{~N}$ \\
\hline $1502+106$ & Quasar & 1.839 & 14.8 & 11.9 & 15.2 & 4.7 & 102.6 & $\mathrm{Y}$ \\
\hline $1510-089$ & Quasar & 0.360 & 20.2 & 16.5 & 20.6 & 3.4 & 101.4 & $\mathrm{Y}$ \\
\hline $1538+149$ & BL Lac & 0.605 & 8.7 & 4.3 & 11.2 & 10.6 & 128.5 & $\mathrm{~N}$ \\
\hline $1606+106$ & Quasar & 1.226 & 17.9 & 24.8 & 18.9 & 2.2 & 71.8 & $\mathrm{~N}$ \\
\hline $1611+343$ & Quasar & 1.397 & 5.7 & 13.6 & 8.0 & 3.0 & 45.9 & $\mathrm{~N}$ \\
\hline $1633+382$ & Quasar & 1.814 & 29.5 & 21.3 & 31.1 & 2.6 & 108.4 & $\mathrm{Y}$ \\
\hline $1637+574$ & Quasar & 0.751 & 10.6 & 13.9 & 11.0 & 4.0 & 75.0 & $\mathrm{~N}$ \\
\hline $1641+399$ & Quasar & 0.593 & 19.3 & 7.7 & 28.0 & 5.1 & 136.4 & $\mathrm{~N}$ \\
\hline $1730-130$ & Quasar & 0.902 & 35.7 & 10.6 & 65.5 & 2.9 & 146.9 & $\mathrm{~N}$ \\
\hline $1739+522$ & Quasar & 1.379 & $\ldots$ & 26.3 & $\ldots$ & $\ldots$ & $\ldots$ & $\mathrm{N}$ \\
\hline $1741-038$ & Quasar & 1.054 & $\ldots$ & 19.5 & $\ldots$ & $\ldots$ & $\ldots$ & $\mathrm{N}$ \\
\hline $1749+096$ & BL Lac & 0.322 & 6.8 & 11.9 & 8.0 & 4.2 & 60.1 & $\mathrm{Y}$ \\
\hline $1803+784$ & BL Lac & 0.684 & 9.0 & 12.1 & 9.4 & 4.5 & 73.4 & $\mathrm{Y}$ \\
\hline $1807+698$ & BL Lac & 0.051 & 0.1 & 1.1 & 1.0 & 45.3 & 50.8 & $\mathrm{~N}$ \\
\hline $1823+568$ & BL Lac & 0.664 & 9.4 & 6.3 & 10.2 & 8.4 & 112.4 & $\mathrm{~N}$ \\
\hline $1828+487$ & Quasar & 0.692 & 13.7 & 5.6 & 19.5 & 7.1 & 135.4 & $\mathrm{~N}$ \\
\hline $1928+738$ & Quasar & 0.302 & 7.2 & 1.9 & 15.0 & 14.8 & 151.2 & $\mathrm{~N}$ \\
\hline $2121+053$ & Quasar & 1.941 & 8.4 & 15.2 & 9.9 & 3.2 & 58.1 & $\mathrm{~N}$ \\
\hline $2134+004$ & Quasar & 1.932 & 2.0 & 16.0 & 8.1 & 0.9 & 14.6 & $\mathrm{~N}$ \\
\hline $2136+141$ & Quasar & 2.427 & 3.0 & 8.2 & 4.7 & 4.5 & 39.9 & $\mathrm{~N}$ \\
\hline $2145+067$ & Quasar & 0.990 & 2.2 & 15.5 & 7.9 & 1.0 & 16.2 & $\mathrm{~N}$ \\
\hline $2200+420$ & BL Lac & 0.069 & 5.0 & 7.2 & 5.4 & 7.5 & 69.7 & $\mathrm{Y}$ \\
\hline $2201+315$ & Quasar & 0.295 & 7.9 & 6.6 & 8.1 & 8.5 & 100.3 & $\mathrm{~N}$ \\
\hline $2223-052$ & Quasar & 1.404 & 14.6 & 15.9 & 14.7 & 3.6 & 85.4 & $\mathrm{~N}$ \\
\hline
\end{tabular}


Table 1. continued.

\begin{tabular}{ccccccccc}
\hline \hline $\begin{array}{l}\text { IAU name } \\
\text { (B1950.0) }\end{array}$ & Class & $z$ & $\beta_{\text {app }}{ }^{1}$ & $\delta_{\text {var }}$ & $\Gamma$ & $\begin{array}{c}\theta \\
(\mathrm{deg})\end{array}$ & $\begin{array}{c}\theta_{\text {src }} \\
(\mathrm{deg})\end{array}$ & LAT-det. $^{2}$ \\
\hline $2227-088$ & Quasar & 1.562 & 8.1 & 15.8 & 10.0 & 3.0 & 54.8 & $\mathrm{Y}$ \\
$2230+114$ & Quasar & 1.037 & 15.4 & 15.5 & 15.4 & 3.7 & 89.9 & $\mathrm{Y}$ \\
$2251+158$ & Quasar & 0.859 & 14.2 & 32.9 & 19.5 & 1.3 & 46.7 & $\mathrm{Y}$ \\
\hline
\end{tabular}

Notes. ${ }^{(1)}$ Fastest non-accelerating, radial apparent speed measured in the source. The speeds differ in some cases from the speeds quoted by Hovatta et al. (2009), partly because Hovatta et al. used results from a preliminary kinematics analysis, and partly because we consider only nonaccelerating, radially moving components in order to estimate the flow speed close to the core, where the mm-wavelength outbursts take place. Due to this, the values of $\Gamma$ and $\theta$ naturally also differ in some cases from those reported by Hovatta et al. ${ }^{(2)}$ Bright $(>10 \sigma)$ LAT-detections during the first three months of science operations (Abdo et al. 2009a).
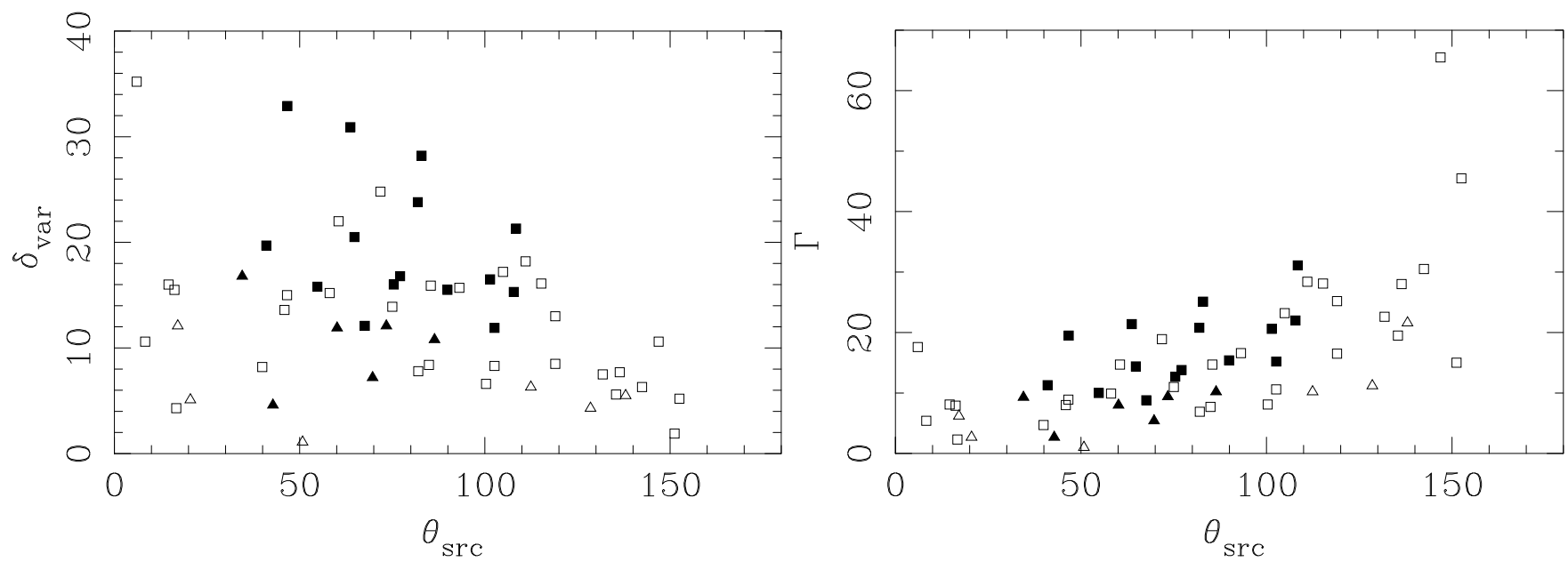

Fig. 2. Left: variability Doppler factor as a function of a comoving frame viewing angle (in degrees) in the MM sample. Squares and triangles denote quasars and BL Lacs, respectively. Filled symbols are LAT-detected sources. Right: jet Lorentz factor as a function of a comoving frame viewing angle in the MM sample.

result regarding higher $\delta_{\text {var }}$ for the LAT-detected blazars to be very robust.

A similar result regarding the high $\delta_{\text {var }}$ of $\gamma$-ray bright sources was found earlier using the less uniform EGRET data (Lähteenmäki \& Valtaoja 2003). The simplest and arguably most likely interpretation is that the $\gamma$-ray bright blazars are indeed systematically more Doppler boosted than the $\gamma$-ray weak ones. This interpretation is compatible with the LAT-detected quasars having faster apparent jet speeds (Lister et al. 2009c), wider apparent jet opening angles (Pushkarev et al. 2009), and higher VLBI brightness temperatures (Kovalev et al. 2009). An alternative interpretation would be that, for some reason, the intrinsic brightness temperature is systematically about a factor of three higher in the LAT-detected blazars than in the non-LAT-detected ones. In the latter case our assumption of a constant limiting brightness temperature would lead to an overestimation of $\delta_{\text {var }}$ in the $\gamma$-ray bright blazars. This alternative explanation would not, however, explain the faster apparent jet speeds or wider apparent jet opening angles of the LAT-detected sources.

The observed difference in the comoving-frame viewing angle distributions between the $\gamma$-ray bright and weak blazars is an unanticipated result. The left panel of Fig. 2 shows that the lack of LAT-detected blazars at large comoving-frame viewing angles can be explained by low Doppler factors of the sources at large $\theta_{\text {src }}$. The beaming model does not, however, explain the lack of LAT-detected sources at small values of $\theta_{\mathrm{src}}$. If this lack is real, it may reflect an intrinsic anisotropy of the $\gamma$-ray emission in the comoving frame of the jet. This would have wide implications for the theoretical models of the high energy emission from blazars, since almost all of these models rely on the assumption that the $\gamma$-ray emission is (nearly) isotropic in the rest frame of the relativistically moving sub-volume (e.g. Dermer 1995). Possible sources of anisotropy in the $\gamma$-ray emission include, for example, anisotropic $\gamma \gamma$ absorption or anisotropic seed photon field for inverse Compton scattering.

The right hand panel of Fig. 2 shows that the non-LATdetected sources at small comoving frame viewing angles have small Lorentz factors, except for B0804+499. This may provide an alternative explanation for the apparent lack of $\gamma$-ray bright sources at small comoving frame viewing angles if the intrinsic $\gamma$-ray luminosity depends on the bulk Lorentz factor in addition to being relativistically beamed. The fact that B0804+499 is not a bright $\gamma$-ray source, despite having a very high Doppler factor and a moderately high Lorentz factor, poses a problem for this explanation, but does not rule it out since the $\gamma$-ray emission may be intermittent (or there may be something unusual in this particular source).

\section{Summary}

We have investigated the connection between the $\gamma$-ray emission of blazars and the intrinsic properties of their parsec-scale radio jets. Our study, based on the 3-month Fermi LAT bright gammaray source list, shows that the $\gamma$-ray bright blazars have higher Doppler factors than the $\gamma$-ray weak ones and confirms the 
earlier results from the EGRET era (e.g., Lähteenmäki \& Valtaoja 2003).

It was also found that the distributions of the viewing angles in the comoving frame of the jet differ significantly between the $\gamma$-ray bright and weak blazars. While the lack of $\gamma$-raydetected sources at large comoving-frame viewing angles can be explained by $\gamma$-ray blazars being more highly beamed, the apparent lack of $\gamma$-ray-detected sources at small comoving-frame viewing angles instead hints of either an intrinsic anisotropy or a Lorentz factor dependence of the $\gamma$-ray emission. Unfortunately, our small sample size and the detection/non-detection nature of the $\gamma$-ray data used in this analysis do not allow firm conclusions to be drawn about the statistical significance of the lack of $\gamma$-raydetected sources at small comoving frame viewing angles if the effects of beaming are taken into account. Since the potential intrinsic emission anisotropy would have particularly important implications for the theoretical models of the $\gamma$-ray production in blazars, further investigation of a larger sample using more extensive $\gamma$-ray flux data is clearly warranted and is being planned on the basis of Fermi 1-year data.

Acknowledgements. We thank Anne Lähteenmäki and the Metsähovi monitoring project for providing unpublished data on two blazars. We thank Charles Dermer for discussions, as well as Ken Kellermann, Andrei Lobanov and Esko Valtaoja for commenting the manuscript. The MOJAVE project is supported under National Science Foundation grant AST-0807860 and NASA Fermi grant NNX08AV67G. T.S. is a research fellow of the Alexander von Humboldt Foundation. T.S. also acknowledges a support by the Academy of Finland grant 120516. D.C.H. was supported by NSF grant AST-0707693. Y.Y.K. is partly supported by the Alexander von Humboldt return fellowship as well as by the Russian Foundation for Basic Research grant 08-02-00545. T.H. acknowledges the support of the Academy of Finland for the Metsähovi observing project. The VLBA is a facility of the National Science Foundation operated by the National Radio Astronomy Observatory under cooperative agreement with Associated Universities, Inc.

\section{References}

Abdo, A. A., Ackermann, M., Ajello, M., et al. 2009a, ApJS, 183, 46 Abdo, A. A., Ackermann, M., Ajello, M., et al. 2009b, ApJ, 700, 597 Atwood, W. B., Abdo, A. A., Ackermann, M., et al. 2009, ApJ, 697, 1071 Blandford, R. D., \& Königl, A. 1979, ApJ, 232, 34

Błażejowski, M., Sikora, M., Moderski, R., \& Madejski, G. M. 2000, ApJ, 545, 107

Bloom, S. D., \& Marscher, A. P. 1996, ApJ, 461, 657

Dermer, C. D. 1995, ApJ, 446, L63

Dermer, C. D., Schlickeiser, R., \& Mastichiadis, A. 1992, A\&A, 256, L27

Ghisellini, G., \& Madau, P. 1996, MNRAS, 280, 67

Ghisellini, G., Tavecchio, F., \& Chiaberge, M. 2005, A\&A, 432, 401

Hartman, R. C., Bertsch, D. L., Bloom, S. D., et al. 1999, ApJS, 123, 79

Hovatta, T., Valtaoja, E., Tornikoski, M., \& Lähteenmäki, A. 2009, A\&A, 494, 527

Jorstad, S. G., Marscher, A. P., Mattox, J. R., et al. 2001a, ApJ, 556, 738

Jorstad, S. G., Marscher, A. P., Mattox, J. R., et al. 2001b, ApJS, 134, 181

Jorstad, S. G., Marscher, A. P., Lister, M. L., et al. 2005, AJ, 130, 1418

Kellermann, K. I., Lister, M. L., Homan, D. C., et al. 2004, ApJ, 609, 539

Kovalev, Y. Y., Kellermann, K. I., Lister, M. L., et al. 2005, AJ, 130, 2473

Kovalev, Y. Y., Aller, H. D., Aller, M. F., et al. 2009, ApJ, 696, L17

Lähteenmäki, A., \& Valtaoja, E. 2003, ApJ, 590, 95

Lister, M. L., Aller, H. D., Aller, M. F., et al. 2009a, AJ, 137, 3718

Lister, M. L., Cohen, M. H., Homan, D. C., et al. 2009b, AJ, 138, 1874

Lister, M. L., Homan, D. C., Kadler, M., et al. 2009c, ApJ, 696, L22

Mannheim, K. 1993, A\&A, 269, 67

Maraschi, L., Ghisellini, G., \& Celotti, A. 1992, ApJ, 397, L5

Mattox, J. R., Bertsch, D. L., Chiang, J., et al. 1993, ApJ, 410, 609

Mattox, J. R., Hartman, R. C., \& Reimer, O. 2001, ApJS, 135, 155

Press, W. H., Flannery, B. P., Teukolsky, S. A., \& Vetterling, W. T. 1992,

Numerical Recipes in FORTRAN 77, 2nd edn. (Cambridge University Press)

Pushkarev, A. B., Kovalev, Y. Y., Lister, M. L., \& Savolainen, T. 2009, A\&A, 507, L33

Readhead, A. C. S. 1994, ApJ, 426, 51

Savolainen, T., Wiik, K., Valtaoja, E., Jorstad, S. G., \& Marscher, A. P. 2002, A\&A, 394, 851

Sikora, M., Begelman, M. C., \& Rees, M. J. 1994, ApJ, 421, 153

Teräsranta, H., Achren, J., Hanski, M., et al. 2004, A\&A, 427, 769

Valtaoja, E., \& Teräsranta, H. 1995, A\&A, 297, L13

von Montigny, C., Bertsch, D. L., Chiang, J., et al. 1995, ApJ, 440, 525 